\title{
Problemorientierung und Methodenpluralismus in den IB
}

Ein Plädoyer für methodischen Nonkonformismus

\begin{abstract}
Dieser Forumsbeitrag plädiert im Sinne einer weiteren Professionalisierung der IB für eine Problemorientierung bei der Methodenwahl und für einen pragmatischen Methodenpluralismus in Forschung und Lehre. Dies setzt gleichzeitig eine Stärkung (der Methodenkompetenz) und Schwächung (des Alleinvertretungsanspruchs bestimmter Methoden) der Methodenfrage voraus. Der Beitrag nimmt zudem eine Auswertung aller bisher in der ZIB erschienenen Aufsätze vor. Diese zeigt, dass der Anteil der methodisch angeleiteten, empirischen Beiträge ebenso wie die Anzahl der theoriegeleiteten Fallstudien und quantitativ statistische Verfahren stetig zunehmen, ohne andere methodische Zugänge und Theoriebeiträge aus dem Blickzu verlieren. Diesen Methodenpluralismus aufgreifend stellt der Beitrag die Entwicklung und Relevanz der Forschungsfrage als zentrales Gütekriterium ins Zentrum, schlägt eine Typologisierung dieser Forschungsfragen vor und nimmt sowohl den Stellenwert methodischer Fragen als auch das Verhältnis von Theorie, Untersuchungsgegenstand und Methode in den IB in den Blick.
\end{abstract}

\section{Einleitung ${ }^{1}$}

Die Internationalen Beziehungen (IB) haben sich in den letzten zwei Jahrzehnten in Deutschland als Teildisziplin der Politikwissenschaften professionalisiert und internationalisiert (Zürn 2003; Deitelhoff/Wolf 2009; Daase 2010). Wie Nicole Deitelhoff und Klaus Dieter Wolf in ihrer Darstellung dieser Entwicklung betonen, beruht diese Professionalisierung primär auf einer kompetenten und kreativen, wenn auch gelegentlich sprunghaften und selektiven Theorieorientierung und einem problemzentrierten Zugang zu den Gegenständen der internationalen Politik, weniger jedoch auf dem fachgerechten Einsatz sozialwissenschaftlicher Methoden. Obwohl die methodisch reflektierte Forschung zugenommen hat (nicht zuletzt dank der Begutachtungsstandards von Fachzeitschriften und Drittmittelgebern) und gelegentlich argumentiert

* Die Autorin und die Autoren dieses Forums sind von der ZIB-Redaktion gebeten worden, sich in ihren Beiträgen mit folgenden fünf Leitfragen auseinanderzusetzen: 1. Welcher Stellenwert sollte methodischen Fragen in den Internationalen Beziehungen (IB) zukommen?; 2. An welchen Kriterien und Traditionen sollten sich die IB in methodischer Hinsicht orientieren?; 3. Was sollten Methoden in den IB leisten?; 4. In welchem Verhältnis stehen Methode und Theorie?; 5. In welchem Verhältnis stehen Methode und Untersuchungsgegenstand? Es stand der Autorin und den Autoren jedoch frei, einzelne Fragen in den Vordergrund zu rücken, andere Fragen nur am Rande zu thematisieren und so eigene Schwerpunkte zu setzen.

1 Wir danken Joachim Blatter, Gabi Schlag sowie der Verfasserin/dem Verfasser des anonymen Gutachtens für wertvolle Anregungen und Kommentare zu diesem Beitrag. 
wird, dass eine weitere Erhöhung der Methodenkompetenz die Gräben innerhalb der Teildisziplin vertiefen könnte, scheint es uns unabdingbar zu sein, im Sinne einer weiteren Professionalisierung des Faches der Methodenfrage einen größeren Stellenwert einzuräumen. Das erfordert nicht nur die Förderung der Methodendebatte in der Forschung, sondern verstärkte Anstrengungen der Methodenausbildung in IB-relevanten Studiengängen (wie es auch in Akkreditierungsverfahren zunehmend gefordert wird). In diesem Forumsbeitrag plädieren wir zum einen für eine konsequente Problemorientierung bei der Methodenwahl und zum anderen für einen bewussten Methodenpluralismus in Forschung und Lehre der IB. Wir werden in einem ersten Abschnitt zunächst auf diese Begriffe und unser Methodenverständnis eingehen, danach in einer Auswertung aller in der Zeitschrift für Internationale Beziehungen (ZIB) erschienenen Aufsätze die relevanten Veröffentlichungstrends in den deutschen IB aus methodischer Sicht nachzeichnen und schließlich in zwei Abschnitten auf die Leitfragen dieses Forums eingehen, indem wir zum einen den Stellenwert methodischer Fragen sowie zum anderen das Verhältnis von Theorie, Untersuchungsgegenstand und Methode in den IB in den Blick nehmen.

\section{Problemorientierung und Methodenpluralismus}

Nachdem die großen ideologischen Debatten um Erkenntnisinteresse und Kritik in der Politikwissenschaft in den 1990er Jahren überwunden zu sein schienen, wurde die Diskussion über das Selbstverständnis der Disziplin nicht mehr politisch, sondern zunehmend (meta-)theoretisch geführt. Eine Debatte zwischen Positivisten und Hermeneutikern, Quantitativen und Qualitativen, Konstruktivisten und Rationalisten entstand, die dort, wo sie konstruktiv ausgetragen wurde, etwa in der Zeitschrift für Internationale Beziehungen, nicht nur produktiv, sondern geradezu identitätsbildend wirkte. Nie war die deutsche IB so vereint wie in der »sagenumwobenen« ZIB-Debatte. Gleichwohl: Die theoretische Spezialisierung und Professionalisierung sowie die Differenzierung in qualitative und quantitative »Schulen« oder »Lager « macht es zunehmend schwierig, Methodendebatten kompetent zu führen. Längst haben sich Subkulturen gebildet, die die Ansätze der anderen schon gar nicht mehr kritisieren, sondern nur noch ignorieren - und das gewiss nicht (immer) in böser Absicht, sondern schlicht aus Unkenntnis (wenn auch häufig mit der Hybris des Besserwissens). Wer vermag denn schon die letzten Entwicklungen der multivariaten Statistik, poststrukturalistischer Diskurstheorie und des agent based modelling kompetent im Verhältnis zueinander zu kommentieren? Aber was bedeutet es für die Einheit des Faches, wenn seine Vertreter und Vertreterinnen die Forschungsleistungen der Kolleginnen und Kollegen nur noch selektiv zur Kenntnis nehmen können? Welche Folgen hat es für das Wissen über internationale Politik, wenn immer kleinere Expertengruppen entstehen, die untereinander nicht mehr kommunizieren und ihre Forschungsergebnisse nicht anerkennen?

Die Lösung dieses Problems liegt unseres Erachtens in der gleichzeitigen Stärkung und Schwächung der Methodenfrage. Stärkung im Sinne einer Stärkung der Metho- 
denkompetenz (bei den Forschenden, Lehrenden und Studierenden), um in der Lage zu sein, andere Ansätze überhaupt zu verstehen und anerkennen zu können. Schwächung im Sinne von Schwächung des Anspruchs auf exklusiven Zugang bestimmter Methoden zu Wirklichkeit und Wahrheit und damit Schwächung ihres Alleinvertretungsanspruchs. Nur in dieser Kombination kann eine methodische Professionalisierung stattfinden, ohne dass das Fach fragmentiert und in methodologische Provinzen zerfällt.

Damit verbunden ist die Notwendigkeit einer konsequenten Problemorientierung des Faches, das eher über empirische und theoretische Problematisierung seine Gegenstände finden sollte als über die methodischen Möglichkeiten ihrer Analyse. Dass problemorientiertes Forschen integrierend wirken kann, haben jüngere Forschungsprogramme wie z.B. zur Regimeforschung oder zur Bürgerkriegsforschung gezeigt. Unter Problemorientierung verstehen wir, dass ausgehend von der theoretischen oder empirischen Fragestellung die Ziele eines Forschungsvorhabens bestimmt werden und daraus abgeleitet seine Methodenwahl vorgenommen wird. ${ }^{2}$ Die Entwicklung und Begründung der Forschungsfrage stellt damit in jedem Fall das zentrale Kriterium für die Güte eines theoriegeleiteten empirischen Forschungsvorhabens dar. Eine reine Methodenfixierung, die einer »Flucht aus der Realität« gleichkommt (Shapiro 2005: 179), ist daher zu vermeiden. Der Frage der Relevanz der Forschungsfrage kommt demgegenüber die entscheidende Rolle zu - sei diese wissenschaftlich oder politisch begründet (vgl. Pierson 2007: 167). Wie wir weiter unten darlegen werden, impliziert dies eine große Bandbreite sinnvoller wissenschaftlicher Forschungsfragen.

Daraus begründet sich auch unser Plädoyer für einen Methodenpluralismus. Unklare Unterscheidungen und Dichotomien, die Gräben zwischen qualitativen und quantitativen, interpretativen und positivistischen Ansätzen, harten und weichen Methoden und ontologischen und epistemologischen Orientierungen vertiefen, sind unseres Erachtens wenig hilfreich. Nach wie vor prägen aber methodologische Glaubensbekenntnisse die Debatte und führen zu einer Aufspaltung der IB in mindestens zwei Kulturen (vgl. Mahoney/Goertz 2006). Sie prägen die Zusammenarbeit in Forschungsverbünden und bei Veröffentlichungen, beeinflussen Gutachterstellungnahmen und Berufungsentscheidungen, und fördern eine Fragmentierung des Faches. Wir plädieren für einen Zugang zu Methodenfragen, der pluralistisch nicht nur in dem Sinne ist, dass er unterschiedliche Ansätze gelten lässt, sondern bewusst Diversität, Entscheidungsfreiheit und Toleranz fördert. Ein solcher Ansatz orientiert sich bei der Wahl des methodischen Vorgehens an der Forschungsfrage und verschließt sich pragmatischen Kombinationen von Methoden, ja selbst von Ontologien und Epistemologien, nicht (vgl. Della Porta/Keating 2008; Monroe 2005).

Die Begriffe »Methoden« und »methodenorientiert« werden vielfältig verwendet. Sie umfassen gängigerweise sowohl Verfahren zur Datenerhebung, Instrumente zur Datenanalyse sowie wissenschaftstheoretische Diskurse. Im Folgenden wollen wir vor allem Instrumente zur Datenanalyse sowie die systematische Reflexion über die

2 Problemorientierung ist mithin nicht identisch mit Problemlösen (oder problem-solving) im Sinne von Cox (1981), sondern eher an das Verständnis von Hellmann (1994) angelehnt. 
Verfahren zur Primärdatenerhebung (wie Interviews oder Archivauswertung) unter den Methodenbegriff subsumieren. Die Methodenwahl der Datenanalyse ist von der Wahl der Datenerhebungsmethode allerdings weitgehend unabhängig. So können beispielsweise die aus Archiven gewonnenen Informationen sowohl für eine Diskursanalyse als auch für eine Qualitative Comparative Analysis (QCA) oder eine Kausalprozessanalyse herangezogen werden und auch als Rohdaten für eine statistische Analyse dienen. Wissenschaftstheoretische Überlegungen sind demgegenüber am besten als Methodologie zu bezeichnen. Systematische (und nicht eklektizistische) Methodenkombinationen sind in vielfältiger Weise möglich: vertikal im Sinne von Methodentriangulation als auch horizontal im Sinne von Methodenparallelismen (Junk 2011).

Sprechen wir von Methoden der IB, beziehen wir uns folglich auf die Methodenorientierung theoriegeleiteter empirischer Forschung und Lehre. Wie Bernhard Kittel (2009) in seiner Auswertung der Aufsätze in vier deutschen politikwissenschaftlichen Fachzeitschriften (Politische Vierteljahresschrift (PVS), Zeitschrift für Politik (ZfP), Zeitschrift für Politikwissenschaft (ZPol) und Zeitschrift für Parlamentsfragen (ZParl)) darlegt, befindet sich die theoriegeleitete, empirische Forschung in der deutschen Politikwissenschaft immer noch in der Minderheit. Das bedeute aber nicht, dass nicht qualitativ hochwertige und methodisch reflektierte Artikel geschrieben, sondern nur, dass diese tendenziell in nicht-deutschen Zeitschriften veröffentlicht würden (Kittel 2009: 595). Da Kittel in seiner Analyse die ZIB nicht berücksichtigt, wollen wir im Folgenden seinen Befund in einer ähnlichen Analyse anhand der in der ZIB erschienenen Aufsätze überprüfen.

\section{Methodische Orientierung von ZIB-Aufsätzen}

Für diesen Beitrag erfolgte eine Auswertung aller als Aufsätze gekennzeichneten wissenschaftlichen Beiträge in der ZIB seit ihrem erstmaligen Erscheinen im Jahr 1994. ${ }^{3}$ In dieser Vollerhebung wurden 118 Aufsätze zunächst danach kodiert, ob sie nach unserer Einschätzung vorrangig empirisch orientiert sind oder sich als vorwiegend theoretische oder methodische Erörterungen einordnen lassen. Weiterhin haben wir die Beiträge, in denen eine Selbstreflexion der Teildisziplin IB im Vordergrund stand, als solche kodiert. Die empirisch orientierten Aufsätze wurden dann weiter nach ihrer Methodenwahl untergliedert, soweit dies möglich war. Wir unterschieden hier zwischen quantitativ-statistischen Verfahren, theoriegeleiteten Fallstudien, Kausalprozessanalysen, QCA (bislang in der ZIB nicht aufgegriffen), inhaltsanalytischen und diskursanalytischen Verfahren, Netzwerkanalysen und reiner Beschreibung (»deskriptiv« und mithin nicht methodisch angeleitet). Mehrfache Einteilungen waren im Falle von methodenkombinatorischen Untersuchungsdesigns möglich.

3 Die Kodierung umfasst somit alle Hefte von 1994/1 bis 2011/1. Wir danken Janusz Biene, Dennis Michels, Wencke Müller, Cara Röhner und Martin Schmetz für die Unterstützung bei dieser Kodierung. 
Wie eine Auswertung der prozentualen Verteilung (Tabelle 1) sowie die korrespondierende Verlaufsdarstellung (Abbildung 1) der Aufsätze der ZIB zeigt, ${ }^{4}$ nimmt der Anteil der methodisch angeleiteten Veröffentlichungen stetig zu. Demgegenüber nimmt der Anteil der reinen Theoriebeiträge und der der disziplinären Selbstreflexionen in der Tendenz ab.

Tabelle 1: Prozentuale Verteilung der ZIB-Aufsätze in Sechsjahreszeiträumen

\begin{tabular}{|l|c|l|c|c|}
\hline Zeitraum & $\begin{array}{l}\text { Methodisch ange- } \\
\text { leiteter Empirie- } \\
\text { beitrag }\end{array}$ & $\begin{array}{l}\text { Theorie- } \\
\text { beitrag }\end{array}$ & $\begin{array}{l}\text { IB-Selbstreflexions- } \\
\text { beitrag }\end{array}$ & $\begin{array}{l}\text { Deskriptiver } \\
\text { Beitrag }\end{array}$ \\
\hline $\mathbf{1 9 9 4 - 1 9 9 9}$ & $45,45 \%$ & $43,18 \%$ & $4,55 \%$ & $6,82 \%$ \\
\hline $\mathbf{2 0 0 0 - 2 0 0 5}$ & $54,17 \%$ & $29,17 \%$ & $2,08 \%$ & $14,58 \%$ \\
\hline $\mathbf{2 0 0 5 - 2 0 1 1}$ & $81,08 \%$ & $16,22 \%$ & $0 \%$ & $2,7 \%$ \\
\hline
\end{tabular}

Abbildung 1: Entwicklung der Publikationspraxis in der ZIB (x-Achse: in Prozent; yAchse: Sechsjahreszeiträume)

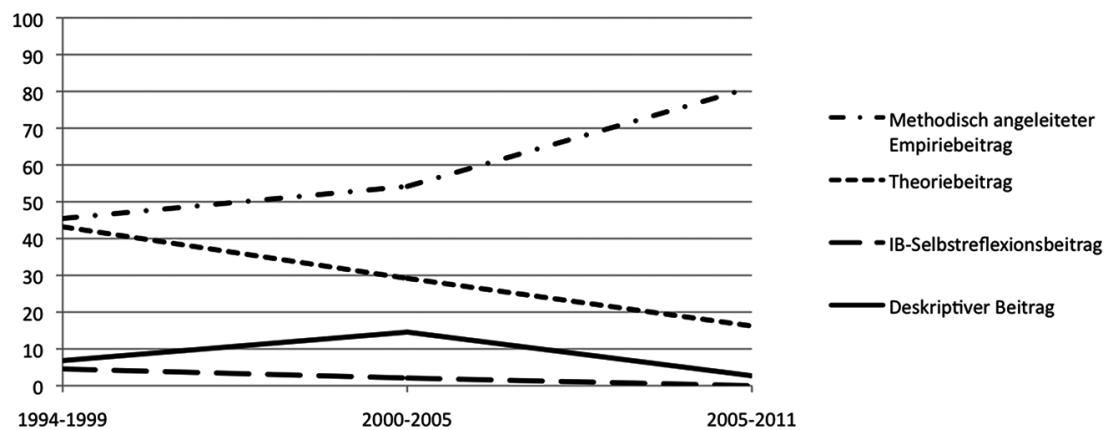

Sieht man sich nun diese methodisch angeleiteten, empirischen Aufsätze näher an (Abbildung 2 und Abbildung 3), so kann man feststellen, dass insbesondere theoriegeleitete Fallstudien sowie quantitativ statistische Verfahren über die Jahre stark zunehmen. Abbildung 3 zeigt jedoch, dass die Aggregation in Sechsjahreszeiträume manche Volatilität verschleiert. Bis auf eine Ausnahme gab es in der ZIB keine Sonderhefte, so dass der Volatilität eher disziplinsoziologische und karrieristische Effekte

4 Kittel (2009) verwendet in seiner Darstellung zum einen Zehnjahreszeiträume und zum anderen jährliche Darstellungen. Unsere Erhebung umfasst die ersten 18 Erscheinungsjahre der ZIB (mit Ausnahme des Heftes 2/2011). Aus Darstellungsgründen haben wir drei Sechsjahreszeiträume aggregiert (Tabelle 1 sowie Abbildung 1 und 2). Um die Volatilität der Methodenkonjunkturen darzustellen, haben wir jedoch eine Abbildung auf Jahresbasis eingestellt (Abbildung 3). 
(kumulierte Einreichungen von verschiedenen Forschungsinstitutionen bspw.) sowie eventuell sogar Präferenzen von Herausgebern zugrunde liegen könnten.

Abbildung 2: Entwicklung der Publikationspraxis in der ZIB (nur methodisch angeleitete empirische Aufsätze; x-Achse: Anzahl der Aufsätze; y-Achse: Sechsjahreszeiträume)

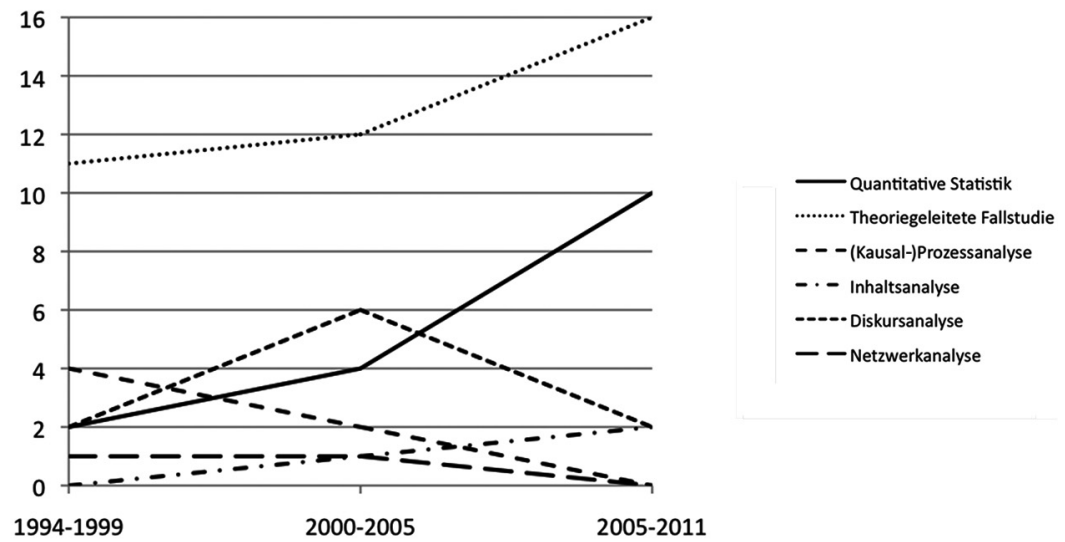

Abbildung 3: Entwicklung der Publikationspraxis in der ZIB (nur methodisch angeleitete, empirische Aufsätze; x-Achse: Anzahl der Aufsätze; y-Achse: Einjahreszeiträume)

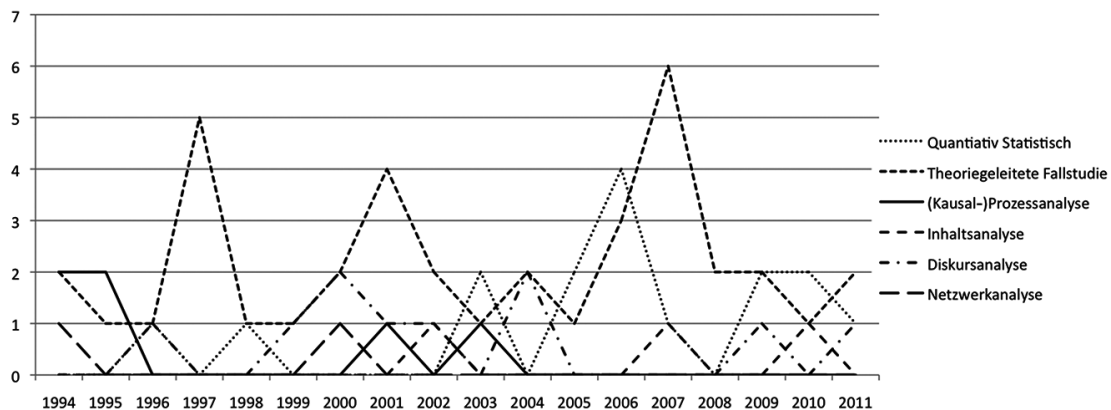

Tendenziell ist bei früheren Aufsätzen der ZIB weniger häufig eine explizite methodische Reflexion zu erkennen, intuitiv-lebensweltlich folgten diese Beiträge jedoch oftmals den Grundsätzen methodisch reflektierter Forschung. Hypothesentests und quantitative Studien, die in den USA das Bild der Politikwissenschaft erheblich prägen, sind auch in der Veröffentlichungspraxis der ZIB zunehmend präsent, aber 
nach wie vor in der Minderheit. Qualitative Studien, häufig hypothesengenerierend und plausibilisierend, sowie theoretische Abhandlungen bilden immer noch das Gros der Veröffentlichungen. Rein methodische Erörterungen sind unter den ZIB-Aufsätzen nicht zu finden. Vergleicht man die ZIB mit den von Kittel (2009) untersuchten vier erwähnten politikwissenschaftlichen Zeitschriften, so orientiert sich erstere qualitativ am ehesten an der PVS. Im Verhältnis zum Durchschnitt der deutschen Publikationsorgane weist die ZIB gemäß der Kriterien von Kittel somit eine höhere wissenschaftliche Qualität und fast keine »impressionistischen Beiträge« oder »sachkundige Erörterungen« auf. Dies dürfte auch der Grund sein, warum laut einer Befragung die ZIB, noch vor der PVS, als »beste deutsche Zeitschrift« in der Politikwissenschaft gilt (Faas/Schmitt-Beck 2009: 637).

\section{Der Stellenwert methodischer Fragen in den Internationalen Beziehungen}

Der Stellenwert methodisch angeleiteter Forschung hat gemäß der vorangegangenen Auswertung der Veröffentlichungspraxis in der ZIB zugenommen. Unserer Ansicht nach wäre es wünschenswert, diesen Trend in dem Sinne zu verstärken, dass sich der Stellenwert methodischer Fragen an der Maßgabe orientiert, dass Methoden lediglich ein Instrumentarium vor allem für die Datenanalyse aber auch für die Datenerhebung zur Verfügung stellen, um die zuvor entwickelten Forschungsfragen zu beantworten. Sie haben daher eine »dienende Funktion« im Forschungsprozess (vgl. Kittel 2009: 580). Die Güte der methodischen Einbettung eines Forschungsvorhabens bemisst sich nach drei Kriterien: erstens, ob die Beantwortung der Forschungsfragen formal-logisch einwandfrei erfolgt; zweitens, ob die Beantwortung der Forschungsfrage in transparenter und nachvollziehbarer und replizierbarer Weise vorgenommen wurde; und, drittens, ob der Anspruch der Methode mit dem nötigen Maß an Bescheidenheit formuliert wurde. Letzteres gilt sowohl hinsichtlich des Generalisierungsanspruchs der Ergebnisse der Forschung als auch hinsichtlich der Abgrenzung von konkurrierenden Ansätzen und etablierten Schulen in den IB.

Methodische Fragen sollten, unserer Ansicht nach, zwar immer der Formulierung einer Forschungsfrage und der Forschungsrelevanz untergeordnet, aber als Bestandteil eines jeden empirischen Forschungsvorhabens ausführlich diskutiert werden. In diesem Sinne kommt ihnen in der IB in Forschung und Lehre in dreierlei Hinsicht ein höherer Stellenwert zu: Erstens wäre es wünschenswert, wenn sich die IB an der Weiterentwicklung der sozialwissenschaftlichen Methodendiskussion aktiver beteiligen und die durch ihre spezifischen Untersuchungsgegenstände bedingte Methodenwahl konstruktiv einbringen würde. So stellen z.B. kollektive Akteure wie Staaten und internationale Organisationen oder die in der Regel kleinen Fallzahlen z.B. von Kriegen, Krisen oder Verhandlungen methodische Probleme dar, die nicht einfach durch einen konsequenten methodologischen Individualismus oder durch die beliebige Erhöhung der Beobachtungen gelöst werden können, sondern kreativere Lösungen verlangen. Zweitens sollte innerhalb der Disziplin nicht nur die Pluralität von Theorien für das Verständnis zentraler Phänomene der internationalen Politik betont, 
sondern auch der Pluralismus der Methoden gefördert werden. Das impliziert drittens, dass neben der Theorieorientierung der Forschung auch die methodische Avanciertheit und Reflexion zu einem Gütekriterium guter empirischer Forschung ausgebaut werden sollte.

Eine unmittelbare Reflexion dieser Entwicklungen wäre auch in der Lehre begrüßenswert. Lehrbücher, die in diese Richtung gehen, gibt es zwar bereits; ${ }^{5}$ an vielen Standorten besteht die Methodenausbildung jedoch lediglich aus einem Grundkurs in Statistik, der nicht selten an fachfremde »Expertinnen und Experten « aus der Soziologie oder gar Ökonomie ausgelagert ist, und im weiteren Verlauf des Studiums keine Rolle mehr spielt. Dadurch verdorren die dort gewonnenen Kompetenzen, weil sie in der wissenschaftlichen Praxis nicht umgesetzt werden. Umfassende und systematische Kurse zu qualitativen Methoden sind bislang die Ausnahme, ebenso wie praktische Übungen zu Methodenwahl und Forschungsdesigns. Eine breite, integrative methodische Grundausbildung, die unsere Gedanken von Problemorientierung und Pluralismus aufgreift und die Forschungs-Design-Entwicklung sowie den gesamten Forschungsprozess ins Zentrum stellt, ist nicht zuletzt für die Gewinnung wissenschaftlichen Nachwuchses zentral. Pluralistisch sollte die Ausbildung allein deshalb sein, um das von Robert Keohane als disziplinäre Schizophrenie bezeichnete Auseinanderklaffen der ontologisch als relevant erachteten Vielfalt der Untersuchungsgegenstände und der epistemologischen Einseitigkeit von manchen Studiengängen durch eine Fixierung entweder auf statistische oder auf interpretative Verfahren zu überwinden (Keohane 2003: 10). Die Vielfalt relevanter Fragestellung erfordert eine Vielfalt nicht nur theoretischer, sondern auch methodischer Ansätze.

Die Berücksichtigung von methodischen Traditionen in Forschung und Lehre ist natürlich weiterhin wichtig, aber eher in einem Sinne der Neuentdeckung und nicht zur Verfestigung von vorher erlangten Positionen. Obwohl es für eine fundierte Methodenausbildung sinnvoll sein kann, die Geschichte der Wissenschaft zu rekonstruieren und sich von den Klassikern der Methodologie und den langen Entwicklungslinien, wie zum Beispiel dem teleologischen, dem kritisch-rationalistischen oder dem hermeneutischen Paradigma inspirieren zu lassen, sind wir der Meinung, dass für aktuelle Methodendebatten ein solches Herangehen häufig in seiner Ausschließlichkeit irreführend ist, da hierbei die Gefahr droht, in der Vergangenheit verhaftet zu bleiben und alte Dichotomien zu reproduzieren. Für uns ist daher eher die Frage nach den aktuellen methodischen Orientierungskriterien für die Entwicklung und Beantwortung von Forschungsfragen von Interesse.

Wenn nun die Zielsetzung der Forschung den Ausgangspunkt einer jeglichen methodischen Debatte bilden soll, ist die Formulierung, Auswahl und Begründung der Forschungsfrage entscheidend. Im Folgenden stellen wir daher fünf aufeinander aufbauende Idealtypen von denkbaren Forschungsfragen vor:

Erstens: Forschungsfragen nach Begriffen, die zu einem Verständnis von Sachverhalten oder einer konzeptionellen Weiterentwicklung beitragen. Beispielsweise die

5 Vgl. Behnke et al. (2010); Blatter et al. (2007); Blatter/Haverland (2011); Brady/Collier (2004); Moses/Knutsen (2007). 
Frage danach, was »Security Governance« eigentlich ist oder wie sich die Begriffe »Krieg« und »Terrorismus« zueinander verhalten. Die Beantwortung der Fragen nach Begriffsverständnissen ist für jede weitere Fragestellung konstitutiv, kann aber auch alleinstehend die Grundlage für ein empirisches Forschungsvorhaben bilden.

Zweitens: deskriptiv-komparative Fragestellungen, die nach Unterscheidungen von Konzeptionen und Fällen fragen. Die Entwicklung von Typologien und Klassifikationen erfüllt eine zentrale Ordnungsfunktion von Wissenschaft und ist eine wichtige Vorstufe kausalanalytischer wie normativer Forschung.

Drittens: kausalanalytische Fragestellungen, welche Ursache-Wirkungszusammenhänge in den Mittelpunkt stellen. Fragen nach Begriffsverständnissen sowie Elementen der Deskription und des Vergleichs sind zwar in jedem Fall Teil eines kausalanalytischen Designs. Gefragt wird hier aber präziser nach den Wirkungsverhältnissen zwischen abhängigen und unabhängigen Variablen und nach notwendigen und hinreichenden Bedingungen und outcomes.

Viertens: normative Analysen, die, vereinfacht gesprochen, eine Bewertungsgrundlage in den Mittelpunkt stellen und somit fragen, auf welcher Grundlage etwas als gut oder schlecht, richtig oder falsch bewertet werden kann. Diese Fragen setzen ebenfalls Fragen des Begriffsverständnisses und komparative Überlegungen voraus. Sie sind also ähnlich wie kausalanalytische Fragestellungen weder konzeptionell voraussetzungslos noch unvergleichbar.

Fünftens: Evaluierungsfragen, die erstens eine normative Diskussion über den Bewertungsmaßstab ins Zentrum stellen und die zweitens und zumeist auf einer normativen Diskussion aufbauend, kausalanalytisch, ko-variant, x-zentriert und oft deskriptiv nach der Wirkung bestimmter Maßnahmen fragen. Insofern können zwei Stränge von Evaluierungsfragen unterschieden werden: Fragen nach der Evaluation der Zielsetzungsprozesse und der Ziele (normative Evaluierungsfragen) und Fragen nach der Evaluation der Zielerreichung (kausalanalytische Evaluierungsfragen).

Diese Idealtypen von, in unseren Augen, legitimen Forschungsfragen decken ein breites Spektrum der Forschung der Internationalen Beziehungen ab. Die Einordnung einer Forschungsfrage hilft, unserer Erfahrung nach, methodische Fragen in der Lehre zu strukturieren sowie sich den Voraussetzungen der eigenen Forschung bewusst zu werden.

\section{Zum Verhältnis von Methode, Theorie und Untersuchungsgegenstand}

Dass die Methode bei der theorieorientierten Forschung nicht den Untersuchungsgegenstand bestimmen dürfe, sondern umgekehrt die empirische Frage die Methodensuche anleiten müsse, ist eine alte Forderung insbesondere qualitativer Forschungsansätze. Aber welche Methode passt zu welchem Untersuchungsgegenstand und wie hängen Empirie und Theorie zusammen? Auch diese methodologischen Fragen werden in den IB gerne als Glaubenskämpfe und vor dem Hintergrund angeblicher Inkommensurabilitäten von ontologischen und epistemologischen Grundüberzeugungen geführt. Aber auch hier scheint uns eine »Abrüstung« in der Auseinandersetzung 
notwendig, um zu einer fruchtbaren Debatte über die komplementären analytischen Potentiale unterschiedlicher Ansätze zu kommen.

Unseres Erachtens ist es wenig hilfreich, in Gary Kings, Robert Keohanes und Sidney Verbas »Designing Social Inquiry« (King et al. 1994) »positivistisches Teufelszeug« zu sehen (gleichgültig wie apodiktisch vieles darin formuliert sein mag) oder dem unter anderem von Dvora Yanow vertretenen »Interpretive Turn« (Yanow/ Schwartz-Shea 2006) generell Unwissenschaftlichkeit vorzuwerfen. Auch wenn man bestimmte Grundannahmen nicht teilt, braucht man nicht zu bezweifeln, dass es sich um seriöse, reflektierte Forschungstraditionen handelt, die nicht nur großen Einfluss in der Politikwissenschaft haben, sondern auch wichtige Aspekte der internationalen Politik beleuchten können. Die großen wissenschaftstheoretischen Fragen nach dem Zugang zur »Wirklichkeit«, nach der Rolle von Kausalität oder dem ontologischen Status von materiellen und ideellen Phänomenen werden aller Voraussicht nach nicht von Theoretikern oder Theoretikerinnen der Internationalen Beziehungen gelöst werden. Die Kämpfe, die über diese Fragen in den IB geführt werden, muten deshalb oft künstlich und nicht selten überzogen an. Das soll nicht heißen, dass sich IB-ler und IB-lerinnen nicht mit wissenschaftstheoretischen Fragen auseinandersetzen sollten. Doch scheint uns diese Auseinandersetzung insbesondere dann Ertrag zu versprechen, wenn nicht danach gefragt wird, ob z.B. Kausalitäten vorliegen oder nicht, sondern welche Fragen man mit einem auf Kausalitätsvorstellungen basierenden Erklärungsansatz stellen und eventuell beantworten kann und welche Perspektiven der Forschung sich eröffnen, wenn man Kausalitätsannahmen abschwächen oder ganz auf sie verzichten würde. Insofern würden wir in Fragen der Epistemologie (und der Ontologie) der IB für einen ähnlichen Nonkonformismus eintreten wie bei der Methodenfrage.

Vielleicht lässt sich die Funktion von Methoden am besten bestimmen, wenn man zunächst das Verhältnis von Theorie und Empirie zugespitzt problematisiert. Die strikte Trennung von Empirie und Theorie ist ein alter positivistischer Glaubenssatz. Nur vor dem Hintergrund dieser Trennung hat es Sinn, den Methoden einen prominenten Status einzuräumen, um die induktive Gewinnung von Theorien aus der Empirie oder die deduktive Überprüfung von Theorien an der Empirie zu gewährleisten. Die qualitativ-interpretative Forschung hat demgegenüber diese Trennbarkeit stets bezweifelt und auf die Theoriegeladenheit der Empirie verwiesen: Es gäbe keine reinen empirischen Daten, aus denen Theorien gewonnen oder mit denen Theorien unabhängig getestet werden könnten. Dies ist ein gewichtiges Argument, trifft aber nicht nur die positivistische (und insbesondere die quantitative) Forschung, sondern auch die qualitative, und das insbesondere dort, wo sie versucht, sich möglichst offen ein empirisches Feld zu erschließen und soziale Prozesse unvoreingenommen zu rekonstruieren. Die Suggestion eines möglichst theoriefreien Zugangs zum »Feld« reproduziert die Trennung von Theorie und Empirie und reduziert damit die analytischen Möglichkeiten, die sich aus einer systematischen Verschränkung von empirischer und theoretischer Arbeit ergeben.

Die Trennbarkeit von Theorie und Empirie infrage zu stellen, heißt nicht, ihre Ununterscheidbarkeit zu behaupten, sondern ihre wechselseitige Verwiesenheit aufein- 
ander zu betonen. Dazu gehört freilich, nicht nur die Theoriegeladenheit der Empirie anzuerkennen, sondern auch, wie Stefan Hirschauer betont, die »Empiriegeladenheit der Theorie« (Hirschauer 2008). Diese Empiriegeladenheit der Theorie tritt nach Hirschauer in doppelter Weise zutage: »als empirische Einbettung von Theorien in historische Kontexte und als ihre Fallbezogenheit, als implizit eingelagerte Empirie« (Hirschauer 2008: 169). Beides ließe sich für die IB in besonders klarer Weise nachzeichnen. Die Einbettung von Theorien in historische Kontexte ist wohl in kaum einer Disziplin so klar (und naheliegend) wie in den Internationalen Beziehungen. Nicht umsonst werden Theorien der Internationalen Beziehungen meist anhand der historischen Entwicklung des 20. Jahrhunderts gelehrt, um die Verwobenheit der internen und externen Geschichte der Disziplin deutlich zu machen (vgl. Abbildung 4). Die Entstehung des Idealismus (und damit der Internationalen Beziehungen als akademisches Fach) ist nicht denkbar ohne den Horror des Ersten Weltkrieges und die Hoffnung auf Frieden durch Recht und internationale Organisationen. Der Aufstieg des Realismus beginnt in der Zwischenkriegszeit und gipfelt im Kalten Krieg. Die Infragestellung des Realismus durch Integrationstheorien und den Institutionalismus ist ohne die europäische Einigung und die Entspannungspolitik der sechziger Jahre nicht denkbar, ebenso wenig wie der Erfolg des Neorealismus ohne die Reagan-Ära und den so genannten Zweiten Kalten Krieg. Auch der Aufstieg des Konstruktivismus fällt nicht zufällig mit dem Ende des Ost-West-Konflikts zusammen und der governance-Ansatz mit der Globalisierung.

Abbildung 4: Externe und interne Geschichte der Internationalen Beziehungen

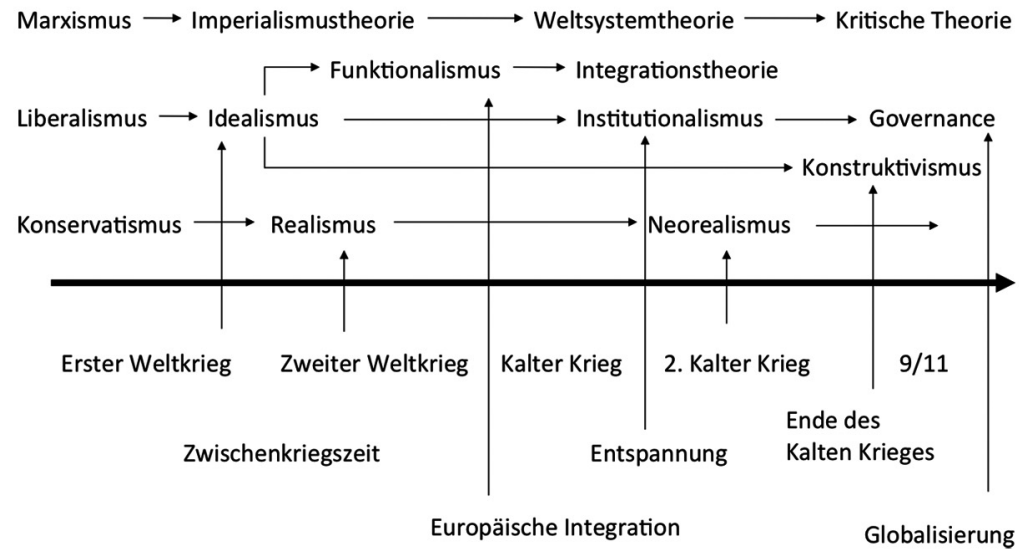

Aber auch die Fallbezogenheit ließe sich in den IB nachweisen und zeigen, wie paradigmatische empirische Fälle in die Theoriebildung eingelassen sind. Denkt man etwa an die Entscheidungen, die zum Ersten Weltkrieg führten, an die Versäumnisse, 
den Aufstieg Hitler-Deutschlands zu verhindern oder die Krisendiplomatie während der Kuba-Krise, dann lässt sich leicht darlegen, wie diese Einzelfälle ganze Generationen von Wissenschaftlerinnen und Wissenschaftlern beschäftigt und die Theoriebildung weit über die jeweilige middle-range theory hinaus geprägt haben.

Wenn man einräumt, dass es ebenso wenig theoriefreie Empirie wie empiriefreie Theorie gibt, dann verliert die Methode ihre herausgehobene Funktion, als gleichsam neutrale Instanz zwischen Theorie und Empirie zu vermitteln. Vielmehr wird sie zu einem Instrument, um theoretisches und empirisches Arbeiten möglichst fruchtbar zu verschränken. Methode garantiert keine »Wahrheit « durch induktives Schließen oder deduktives Testen, sondern ermöglicht eine produktive Wechselwirkung zwischen Empirie und Theorie. Letztlich ist es diese Wechselwirkung, die wissenschaftliche Erkenntnis auch in den Internationalen Beziehungen ausmacht.

\section{Fazit}

In diesem Forumsbeitrag plädierten wir zum einen für eine Problemorientierung der Forschung, die von der Zielbestimmung der Fragestellung auf das angemessene methodische Vorgehen schließt. Zum anderen forderten wir einen Methodenpluralismus in Forschung und Lehre der IB, um der weiteren Fragmentierung der Disziplin entgegenzuwirken und die inhaltlichen Forschungsstränge wieder dichter zusammenzuführen. Der Beitrag plädiert damit für einen methodischen Nonkonformismus, der notabene nicht bedeutet, dass Methoden vernachlässigt werden können. Ganz im Gegenteil sollte man Methodenfragen verstärkt Aufmerksamkeit schenken. Aber die Wahl von Methoden und ihr Wahrheitsanspruch sollte pragmatisch gehandhabt und der jeweils leitenden Forschungsfrage untergeordnet werden.

Eine Auswertung der bisher erschienenen Aufsätze in der ZIB ergab zweierlei: Zum einen nimmt der Anteil der methodisch angeleiteten, empirischen Beiträge stetig zu; zum anderen steigt auch die Anzahl der theoriegeleiteten Fallstudien sowie der Beiträge, die quantitativ statistische Verfahren verwenden, deutlich, ohne dass dies jedoch zu sehr auf Kosten anderer Methoden und der theoretischen Beiträge geht. Insofern bietet die ZIB ein geeignetes Forum, auch über die komplementären Fähigkeiten unterschiedlicher Methoden zu reflektieren.

Auch dieser Beitrag muss sich freilich die Frage nach Problemorientierung und Pluralismus stellen. Als methodische Selbstvergewisserung versuchte er das Problem zu lösen, das durch die zunehmende Spezialisierung und Professionalisierung in Methodenfragen auf die IB zukommt. Der Vorschlag, Methoden gleichzeitig mehr und weniger Bedeutung zu schenken, sie in Forschung und Lehre nachdrücklicher zu thematisieren und sie gleichzeitig weniger restriktiv und mit dem Anspruch auf Alleingültigkeit zu vertreten, scheint uns am ehesten den vielfältigen Ansprüchen an die wissenschaftliche Praxis gerecht zu werden. Die Fragen für dieses Forum, so schien uns, zielten allerdings auf eine stärkere methodische Festlegung. Wir haben versucht, einer solchen Festlegung so weit wie möglich zu widerstehen und eine nonkonformistische Haltung einzunehmen. Letztlich stimmen wir nämlich Paul Feyerabend zu, 
der schrieb: „Science is an essentially anarchic enterprise: theoretical anarchism is more humanitarian and more likely to encourage progress than its law-and-order alternatives « (Feyerabend 1975: 9).

\section{Literatur}

Behnke, Joachim/Baur, Nina/Behnke, Nathalie 2010: Empirische Methoden der Politikwissenschaft, 2. erw. Auflage, Paderborn.

Blatter, Joachim/Haverland, Markus 2011: Designing Case Studies: Explanatory Approaches in Small-N Research, Basingstoke, i.E.

Blatter, Joachim/Janning, Frank/Wagemann, Claudius 2007: Qualitative Politikanalyse. Eine Einführung in Methoden und Forschungsansätze, Wiesbaden.

Brady, Henry/Collier, David 2004: Rethinking Social Inquiry: Diverse Tools, Shared Standards, Lanham, MD.

Cox, Robert W. 1981: Social Forces, States and World Orders: Beyond International Relations Theory, in: Millennium: Journal of International Studies 10: 2, 126-155.

Daase, Christopher 2010: Theorie der Internationalen Beziehungen, in: Gerlach, Irene/Jesse, Eckard/Kneuer, Marianne/Werz, Nikolaus (Hrsg.): Politikwissenschaft in Deutschland, Baden-Baden, 317-338.

Deitelhoff, Nicole/Wolf, Klaus Dieter 2009: Der Widerspenstigen Selbst-Zähmung? Zur Professionalisierung der Internationalen Beziehungen in Deutschland, in: Politische Vierteljahresschrift 50: 3, 451-475.

Della Porta, Donatella/Keating, Michael (Hrsg.) 2008: Approaches and Methodologies in the Social Sciences: A Pluralist Perspective, Cambridge.

Faas, Thorsten/Schmitt-Beck, Rüdiger 2009: Die Politische Vierteljahresschrift im Urteil der Profession. Ergebnisse einer Umfrage unter den Mitgliedern der DVPW, in: Politische Vierteljahresschrift 50: 3, 627-645.

Feyerabend, Paul 1975: Against Method: Outline of an Anarchistic Theory of Knowledge, London.

Hellmann, Gunther 1994: Für eine problemorientierte Grundlagenforschung. Kritik und Perspektiven der Disziplin »Internationale Beziehungen« in Deutschland, in: Zeitschrift für Internationale Beziehungen 1: 1, 65-90.

Hirschauer, Stefan 2008: Die Empiriegeladenheit von Theorien und der Erfindungsreichtum der Praxis, in: Kalthoff, Herbert/Hirschauer, Stefan/Lindemann, Gesa (Hrsg.): Theoretische Empirie. Zur Relevanz qualitativer Forschung, Frankfurt a. M., 165-187.

Junk, Julian 2011: Method Parallelization and Method Triangulation: Method Combinations in the Analysis of Humanitarian Interventions, in: German Policy Studies 7: 3, 83-116.

Keohane, Robert O. 2003: Disciplinary Schizophrenia: Implications for Graduate Education in Political Science, in: Qualitative Methods 1: 1, 9-12.

King, Gary/Keohane, Robert O./Verba, Sidney 1994: Designing Social Inquiry: Scientific Inference in Qualitative Research, Princeton, NJ.

Kittel, Bernhard 2009: Eine Disziplin auf der Suche nach Wissenschaftlichkeit. Entwicklung und Stand der Methoden in der deutschen Politikwissenschaft, in: Politische Vierteljahresschrift 50: 3, 577-603.

Mahoney, James/Goertz, Gary 2006: A Tale of Two Cultures: Contrasting Quantitative and Qualitative Research, in: Political Analysis 14: 2, 227-249.

Monroe, Kristen R. (Hrsg.) 2005: Perestroika! The Raucous Rebellion in Political Science, New Haven, CT.

Moses, Jonathan W./Knutsen, Torbjörn L. 2007: Ways of Knowing: Competing Methodologies and Methods in Social and Political Research, Basingstoke. 
Pierson, Paul 2007: The Costs of Marginalization: Qualitative Methods in the Study of American Politics, in: Comparative Political Studies 40: 2, 146-169.

Shapiro, Ian 2005: The Flight from Reality in the Social Sciences, New Haven, CT.

Yanow, Dvora/Schwartz-Shea, Pregrine 2006: Interpretation and Method: Empirical Research Methods and the Interpretive Turn, Armonk, NY.

Zürn, Michael 2003: Die Entwicklung der Internationalen Beziehungen im deutschsprachigen Raum nach 1989, in: Hellmann, Gunther/Wolf, Klaus Dieter/Zürn, Michael (Hrsg.): Die neuen Internationalen Beziehungen. Forschungsstand und Perspektiven in Deutschland, Baden-Baden. 